\title{
Soft-Tissue Myoepithelioma of the Retroperitoneal Space Mimicking a Pancreatic Tumor: A Case Report and Literature Review
}

\author{
Andrei Nikiforchin ${ }^{\mathrm{a}}$, Ekaterina Baron ${ }^{\mathrm{a}}$, Michelle Sittig ${ }^{\mathrm{a}}$, \\ Vadim Gushchin ${ }^{\mathrm{a}, \mathrm{b}}$
}

\begin{abstract}
Soft-tissue myoepithelioma (STM) is an extremely rare benign tumor with predominant occurrence in head, neck, pelvic girdle and limbs. These tumors lack specific clinical and morphological features and can easily be confused with more common neoplasms. It may lead to incorrect diagnosis and management. Here, we present a clinical case of a young man with retroperitoneal STM which simulated a pancreatic tumor and required a distal pancreatectomy. Performed literature review highlights current data about clinical, morphologic, immunohistochemical and genetic evaluation, treatment and prognosis of STM.
\end{abstract}

Keywords: Soft-tissue myoepithelioma; Myoepithelial tumor; Retroperitoneal neoplasm; Morphology; Immunohistochemical analysis; Gene rearrangements

\section{Introduction}

Myoepithelioma is a rare benign tumor originating from ectodermally derived myoepithelial cells, which are mostly represented in exocrine glands between the acinar sinus and intercalated duct $[1,2]$. Typically it occurs in salivary, sweat, lacrimal and mammary glands, but incidentally can be found in soft tissues, bones and viscera [3-5]. When this neoplasm presents in soft tissues close to organs, it might be incorrectly diagnosed as another primary tumors, which are more common for those locations. It leads to misdiagnosis contributing to errors in appropriate treatment. We report a clinical case of a retroperitoneal soft-tissue myoepithelioma (STM) which simulated a primary pancreatic tumor.

Manuscript submitted December 20, 2019, accepted January 4, 2020

${ }^{a}$ Institute for Cancer Care, Mercy Medical Center, Baltimore, MD 21202, USA ${ }^{b}$ Corresponding Author: Vadim Gushchin, Institute for Cancer Care, Mercy Medical Center, 227 Saint Paul Place, Weinberg Building, 4th Floor, Baltimore, MD 21202, USA. Email: vgushchin@mdmercy.com

doi: https://doi.org/10.14740/jmc3407

\section{Case Report}

A 39-year-old Caucasian man presented with a 1-month history of decreased appetite, mild abdominal pain and palpable epigastric abdominal mass. He was a lifelong nonsmoker with no significant past medical or family history. Computed tomography $(\mathrm{CT})$ scan revealed a large epigastric mass $(14 \times 11$ $\times 11 \mathrm{~cm}$ ) growing from the pancreas with dislocated stomach, duodenum and left-sided portal hypertension (Fig. 1). Positron emission tomography-CT (PET-CT) with F-18 fluorodeoxyglucose (F-18 FDG) showed patchy tracer uptake of the tumor located in the pancreatic body and tail (Fig. 2). There was no evidence of regional or distant disease and all blood tests were within normal limits. Upper endoscopy with transluminal ultrasound-guided biopsy was performed. Final pathology suggested pancreatic myoepithelioma.

The patient underwent distal pancreatectomy. Intraoperatively, there was tumor compression of the superior mesenteric vein (SMV) and splenic vein junction with left-sided portal hypertension and severe inflammatory reaction of adjacent tissues. These findings were suspicious for malignant behavior of the neoplasm and led to technical difficulties. Complete removal of the mass required distal pancreatectomy and splenectomy, with estimated blood loss $2,800 \mathrm{~mL}$. The patient recovered uneventfully and was discharged on the seventh postoperative day.

Postoperative pathology revealed a well-defined multinodal neoplasm with chondroid stroma and monotonous spindle cells having a co-expression of CAM5.2, smooth muscle actin (SMA) and S-100 protein (Figs. 3-6). The retroperitoneal STM was adjacent to pancreas without true invasion. The multidisciplinary tumor board recommended a $3-6$ months follow-up with imaging for 2 years and annual examination for the next 3 years.

At 15 months follow-up, the patient is asymptomatic without signs of malnutrition, malabsorption, or recurrence.

\section{Discussion}

This patient had retroperitoneal STM which was presumed to be a primary pancreatic tumor. Generally, myoepithelioma is a rare glandular tumor that is typically found in salivary glands 


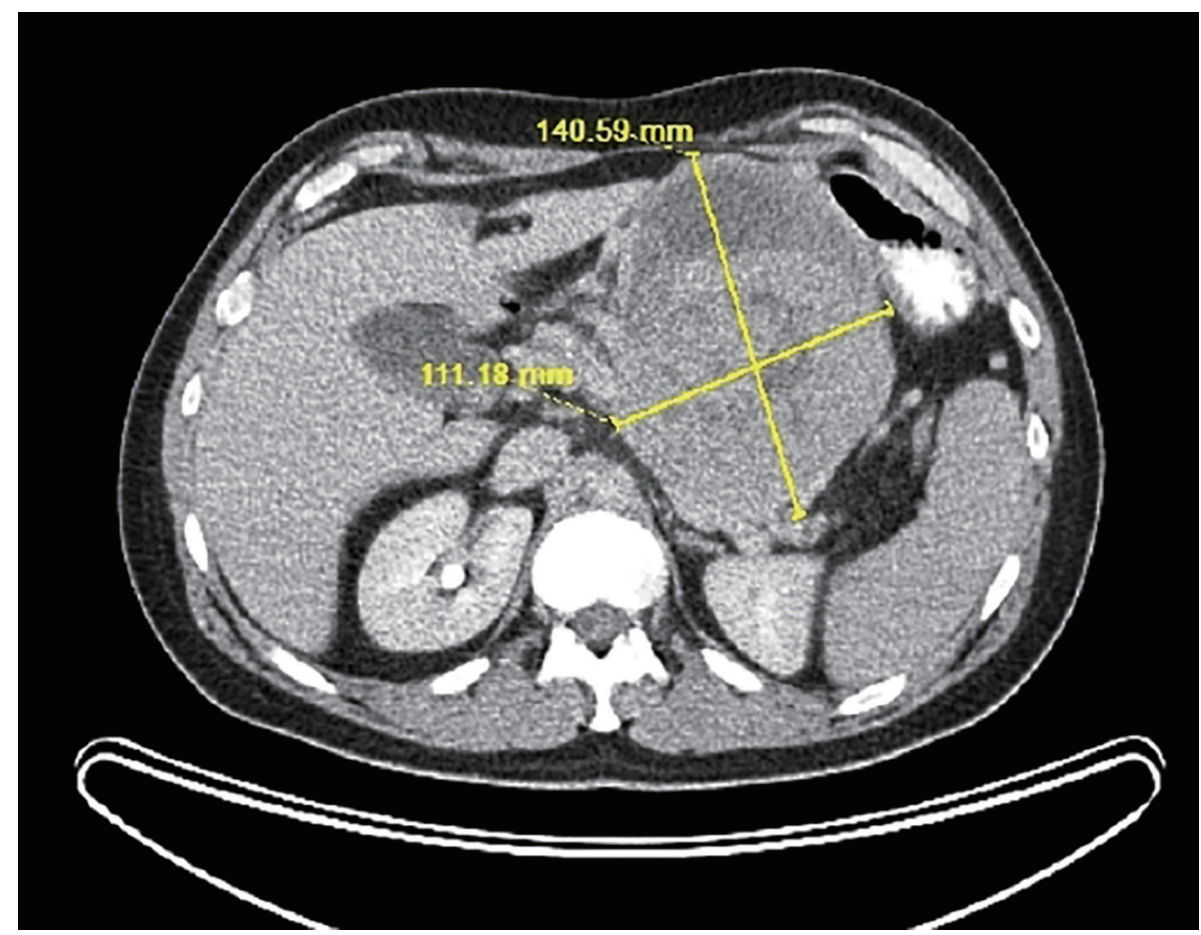

Figure 1. Abdominal CT scan of the left upper quadrant heterogeneous mass measuring $13.6 \times 11.0 \times 11.0 \mathrm{~cm}$. The stomach is displaced laterally. CT: computed tomography.

$[2,5,6]$. Rarely it occurs in soft tissues with predominant growth in head and neck, pelvic girdle and limbs, where it usually appears as a palpable subcutaneous or subfascial painful or painless mass $[3,7]$. There are few reported cases of non- typical sites like orbit, mediastinum, abdominal wall, mesentery and ovary [8-12]. Retroperitoneal space is an extremely uncommon localization for this tumor, described in single publications [13].

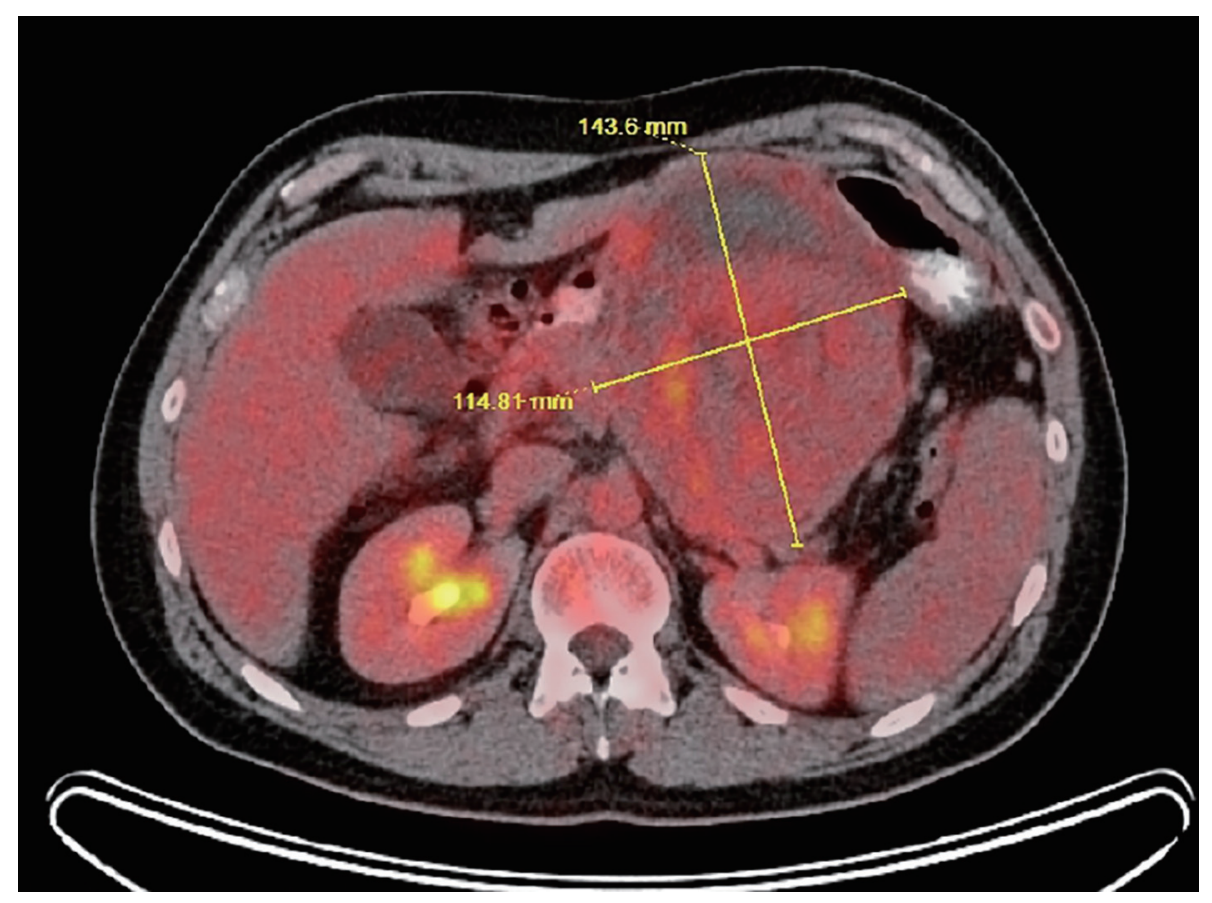

Figure 2. PET-CT scan shows $14.6 \times 10.5 \times 10.2 \mathrm{~cm}$ mass with cystic and solid regions and patchy tracer uptake (max SUV is 5.4). PET-CT: positron emission tomography-computed tomography; SUV: standardized uptake values. 


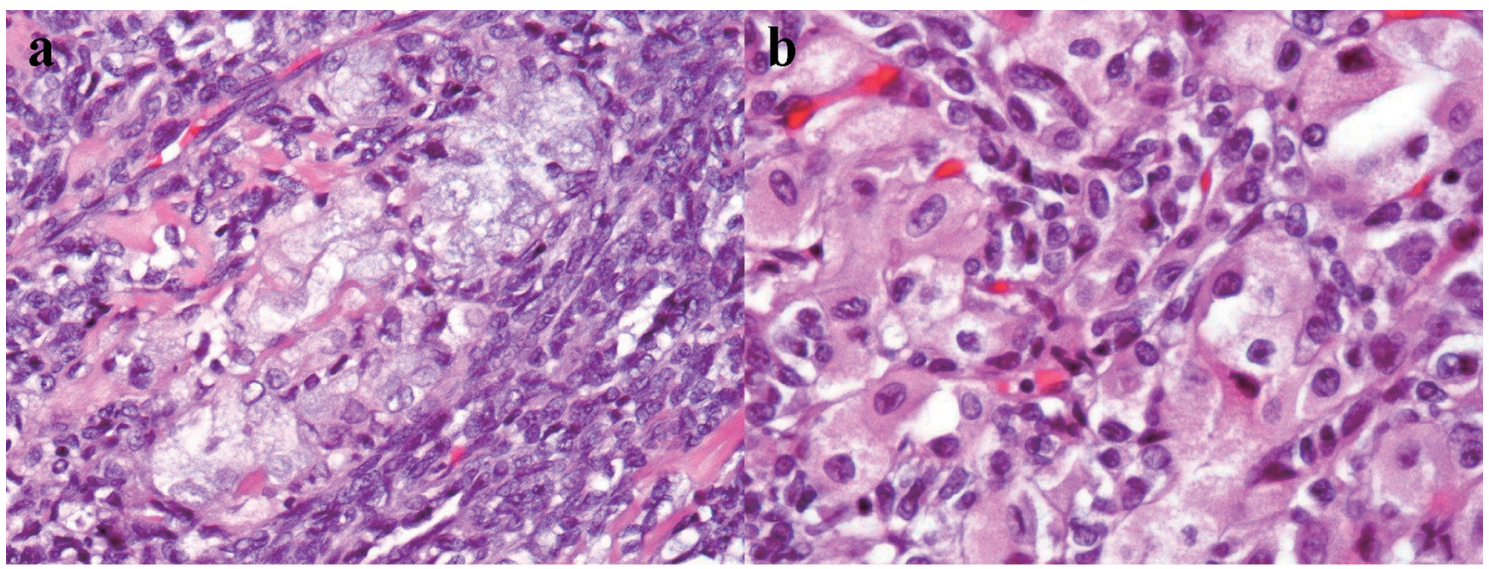

Figure 3. Microscopic examination shows chondroid stroma with monotonous spindle cells with predominantly retiform features, focal plasmacytoid cell morphology and cartilaginous differentiation (H\&E, a: $\times 200$ magnification; b: $\times 400$ magnification). H\&E: hematoxylin \& eosin.

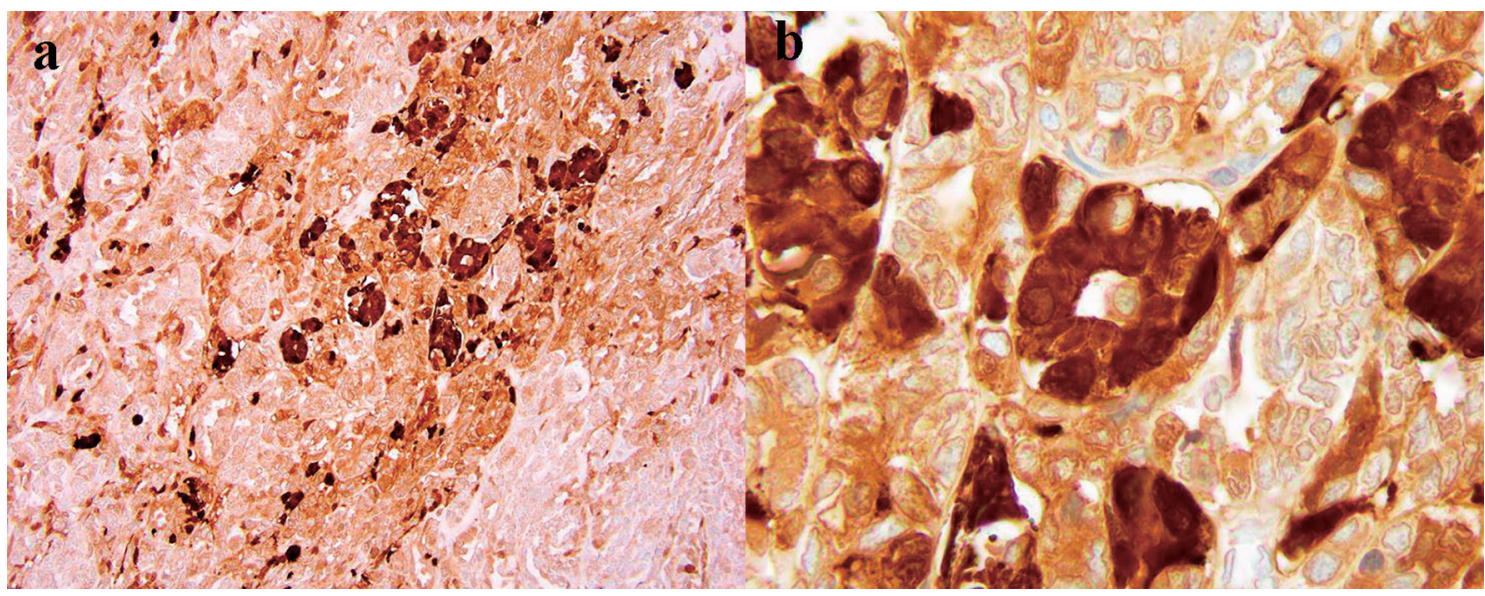

Figure 4. The diffuse expression of S-100 protein by tumor cells (a: $\times 100$ magnification; $\times 400$ magnification).

The diagnosis of STM may be difficult due to lack of specific clinical and imaging guidelines. Some authors consider that moderate enhancement and well-defined cystic compo- nent of tumor on both $\mathrm{CT}$ and magnetic resonance imaging may be representative imaging characteristics for STM [10]. This is consistent with CT and PET-CT findings in our clinical

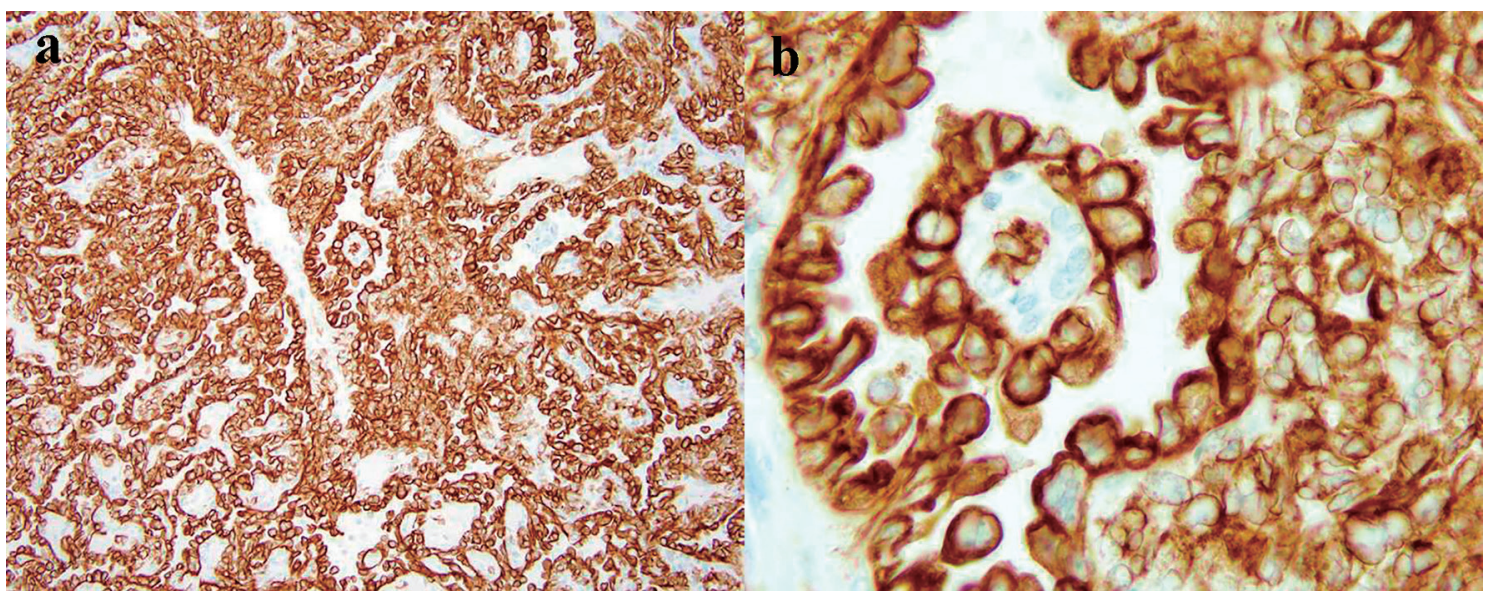

Figure 5. The intensive diffuse CAM5.2 expression of tumor cells (a: $\times 40$ magnification; $b: \times 400$ magnification). 


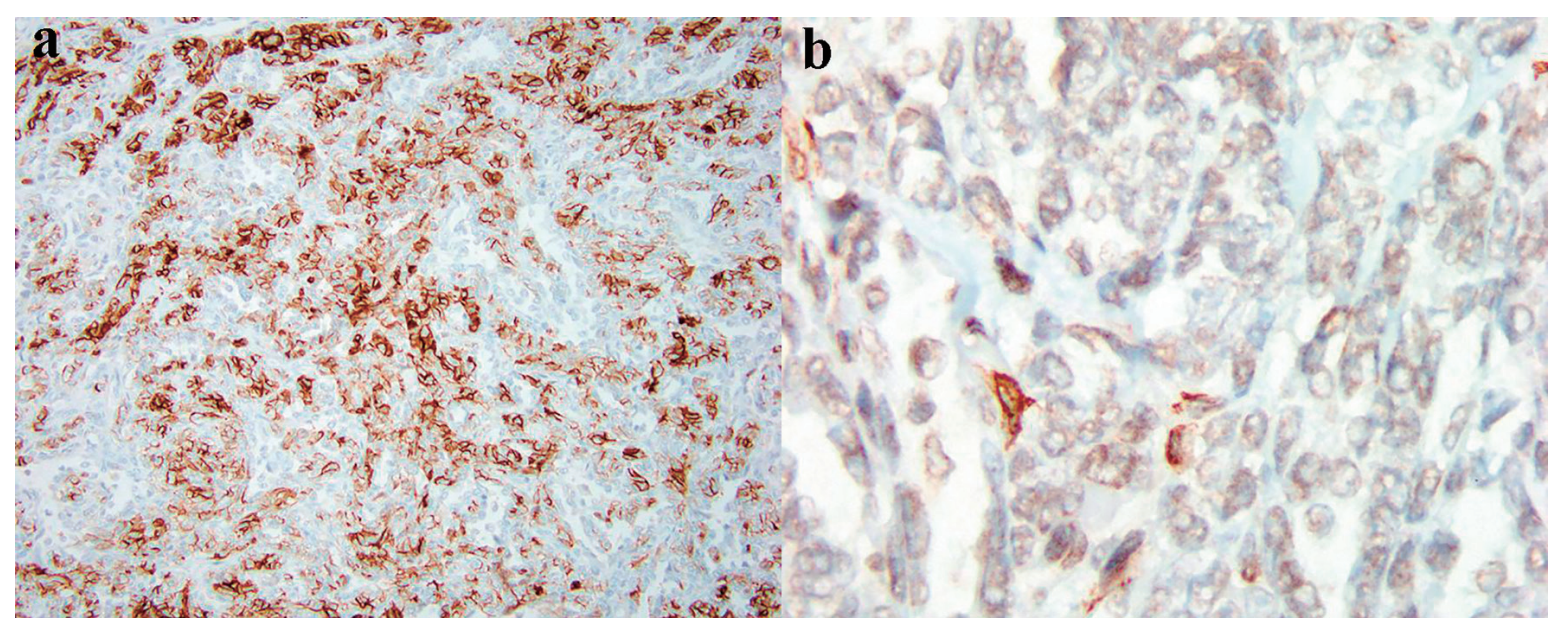

Figure 6. The diffuse expression of SMA by tumor cells ( $\mathrm{a}: \times 40$ magnification; $\mathrm{b}: \times 200$ magnification). SMA: smooth muscle actin.

case (Figs. 1 and 2). However, these traits are not truly specific and seen in a variety of other neoplasms, such as pancreatic and gastrointestinal stromal tumors (GISTs), as well as in sarcomas. Thus, a confirmed histopathologic diagnosis prior to treatment is needed to best guide clinical management.

Core needle-guided biopsy is the preferred diagnostic method for pancreatic tumors due to its high accuracy and low complication rate [14]. The patient underwent upper endoscopy with transluminal ultrasound-guided biopsy of the tumor, which surprisingly confirmed benign myoepithelioma. Nevertheless, this preliminary diagnosis of STM seemed unlikely due to the uncommon localization and well-known difficulties of morphological evaluation.

Pathology examination of STM is challenging owing to microscopical diversity in cell morphology and types of stroma [3, 12]. However, several studies showed that well-circumscribed margins and multinodular growth of spindled and plasmacytoid cells with eccentric nuclei in myxoid, hyalinized or chondroid stroma could be hallmarks for this benign tumor $[3,5,15]$. In contrast, myoepithelial carcinomas are characterized by infiltrative or destructive growth pattern and large cells with irregular and enlarged nuclei embedded in abundant extracellular myxoid matrix. Focal necrosis and large polyhedral cells can also be seen [16]. In the demonstrated case, biopsy revealed a monotonous neoplasm with myoepithelial-type cells and structure that suggested pancreatic myoepithelioma. Postoperative pathology confirmed the diagnosis of STM after assessment of a tumor with well-defined margins and multifocal growth of monotonous spindle cells with focal plasmacytoid morphology and cartilaginous differentiation (Fig. 3a, b). There was no histological evidence indicating that the STM originated from the pancreas.

Considering the challenges of STM microscopic evaluation, the immunohistochemical (IHC) analysis is critical for proper diagnosis. In two large studies, the most sensitive markers for these tumors were keratin $\mathrm{AE} / 1-\mathrm{AE} / 3$ or pan-keratin (expressed in $93 \%$ of cases), vimentin $(100 \%), \mathrm{S}-100$ protein (85-87\%), calponin (86-100\%), epithelial membrane antigen (EMA, 63\%) and glial fibrillary acidic protein (GFAP, 46\%)
$[3,17]$. Other authors described co-expression of additional markers: cytokeratin CAM5.2 (93-100\%), SMA (36-64\%), muscle-actin-specific monoclonal antibody (HHF-35, 20$60 \%)$ and p63 (7-45\%) $[3,15,18,19]$. In our case, immunohistochemistry analysis showed diffuse tumor expression of CAM5.2, SMA and S-100 protein (Figs. 4-6), but did not show expression of CD117 (c-kit) and CD34, which is consistent with IHC criteria of STM diagnosis. The two last markers are also important for differential diagnosis, as their presence is quite specific for GISTs [20].

Currently, the role of genetic testing in STM diagnosis is disputable although its molecular profile has been a subject of investigation over the last decade. The most recent data state that up to $45 \%$ of STM demonstrate Ewing sarcoma region 1 (EWSR1) gene rearrangement by fluorescence in situ hybridization analysis $[15,17,21,22]$. Skalova et al have found the EWSR1 translocations to be present in 39\% (20/51) of clear cell myoepithelial carcinoma specimens, but their cohort included only salivary gland carcinomas [23]. In case of EWSR1-negative STM 38\% harbor the rearrangement of pleomorphic adenoma gene 1 (PLAG1) and a small subset of STM $(9 \%)$ show fused in sarcoma (FUS) gene alterations [24, 25]. Thus, today the data are not sufficient to consider any genetic finding as a reliable diagnostic tool for STM [15-17, 26]. Therefore, our genetic counselors decided not to perform molecular testing in this patient.

The pretreatment diagnosis of STM in our clinical case remained controversial even after testing, since we saw a combination of benign (absence of invasion and metastases on CT, biopsy results) and malignant (tumor size, left-sided portal hypertension, patchy F-18 FDG uptake on PET-CT) clinical signs. Eventually, this case was considered as a pancreatic potentially malignant myoepithelioma requiring surgery. The tumor compression of SMV and splenic vein junction with left-sided portal hypertension seen during the surgery was suspicious for malignant behavior. Also, a severe peritumoral inflammatory reaction complicated visualization and made it impossible to determine whether or not the tumor was originally pancreatic. Considering the intraoperative findings and suspected malig- 
nant pattern of tumor, the patient underwent distal pancreatectomy with splenectomy and lymph node dissection.

Surgical resection with complete removal (R0 status) is a preferable treatment option of STM. Incomplete resection significantly increases risks of recurrence even in benign myoepitheliomas $[3,12]$. The vast majority of these tumors show a favorable prognosis and low incidence of distant metastases $[3,15]$. Nonetheless, in rare cases STM can transform into malignant myoepithelial carcinoma when untreated [27, 28]. Behavior of myoepithelial carcinoma is much more aggressive: the local recurrence rate is $39-42 \%$ and development of metastatic disease occurs in up to $52 \%$ of patients $[3,15,18]$. The tumor of the described patient was removed completely and assessed lymph nodes were negative for tumor tissue. Fifteen months after treatment, the patient had no complaints and signs of disease recurrence.

\section{Conclusion}

This case report demonstrates diagnostic and treatment challenges of a rare tumor with uncommon localization - retroperitoneal STM. We believe our clinical experience will help guide clinicians to the appropriate treatment and reduce the risk of undesirable patient outcomes.

\section{Acknowledgments}

The authors are tremendously grateful to Dr. Kimberley D. Studeman (Department of Pathology, Mercy Medical Center, Baltimore, MD, USA) for reviewing the slides and providing the images.

\section{Financial Disclosure}

The authors have no grants and financial support to disclose.

\section{Conflict of Interest}

The authors declare they have no competing interests.

\section{Informed Consent}

The signed informed consent was obtained from the patient before conducting the study.

\section{Authors Contributions}

Andrei Nikiforchin and Vadim Gushchin had the idea for the concept of this manuscript. Andrei Nikiforchin designed the paper, made literature search and wrote the first manuscript draft. Ekaterina Baron performed a review and editing of the manuscript. Michelle Sittig reviewed the manuscript, and ed- ited it for English, stylistics and grammar. Vadim Gushchin performed the final review of the manuscript. All authors contributed to the review and amendments of the manuscript for important intellectual content and approved the final version for submission.

\section{Data Availability}

The authors declare that data supporting the findings of this study are available within the article.

\section{References}

1. El-Naggar AK, Chan JKC, Takata T, Grandis JR, Slootweg PJ. The fourth edition of the head and neck World Health Organization blue book: editors' perspectives. Hum Pathol. 2017;66:10-12.

2. Barnes L, Appel BN, Perez H, El-Attar AM. Myoepithelioma of the head and neck: case report and review. J Surg Oncol. 1985;28(1):21-28

3. Hornick JL, Fletcher CD. Myoepithelial tumors of soft tissue: a clinicopathologic and immunohistochemical study of 101 cases with evaluation of prognostic parameters. Am J Surg Pathol. 2003;27(9):1183-1196.

4. Kurzawa P, Kattapuram S, Hornicek FJ, Antonescu CR, Rosenberg AE, Nielsen GP. Primary myoepithelioma of bone: a report of 8 cases. Am J Surg Pathol. 2013;37(7):960-968.

5. Thway K, Fisher C. Myoepithelial tumor of soft tissue: histology and genetics of an evolving entity. Adv Anat Pathol. 2014;21(6):411-419.

6. Jo VY, Fletcher CD. WHO classification of soft tissue tumours: an update based on the 2013 (4th) edition. Pathology. 2014;46(2):95-104.

7. Lee JR, Georgi DE, Wang BY. Malignant myoepithelial tumor of soft tissue: a report of two cases of the lower extremity and a review of the literature. Ann Diagn Pathol. 2007;11(3):190-198.

8. Hayward DM, Yoo D, Lee JM, Wild E, Prabhu VC. Myoepithelioma of the orbital apex and middle cranial fossa: case report and review of the literature. Neuroophthalmology. 2014;38(1):14-20.

9. Hashmi AA, Khurshid A, Faridi N, Edhi MM, Khan M. A large mediastinal benign myoepithelioma effacing the entire hemithorax: case report with literature review. Diagn Pathol. 2015;10:100.

10. Guo L, Zhou F, Zhang N, Dai H, Zeng X, Gong H. Myoepithelioma of the lateral abdominal wall: A case report. Medicine (Baltimore). 2018;97(27):e11209.

11. Houghton OP, Sumathi VP, Loyson SA, McCluggage WG. Myoepithelioma of the ovary: first reported case. Int J Gynecol Pathol. 2014;33(2):191-196.

12. Thway K, Noujaim J, Thomas DM, Fisher C, Jones RL. Myoepithelial Carcinoma of the Paracecal Mesentery: Aggressive Behavior of a Rare Neoplasm at an Unusual Anatomic Site. Rare Tumors. 2017;9(1):6504. 
13. Burke T, Sahin A, Johnson DE, Ordonez NG, Mackay B. Myoepithelioma of the retroperitoneum. Ultrastruct Pathol. 1995;19(4):269-274.

14. Larghi A, Verna EC, Stavropoulos SN, Rotterdam H, Lightdale CJ, Stevens PD. EUS-guided trucut needle biopsies in patients with solid pancreatic masses: a prospective study. Gastrointest Endosc. 2004;59(2):185-190.

15. Jo VY, Fletcher CD. Myoepithelial neoplasms of soft tissue: an updated review of the clinicopathologic, immunophenotypic, and genetic features. Head Neck Pathol. 2015;9(1):32-38.

16. Ni H, Zhao PY, Wang XT, Xia QY, Wang X, Wu N, Shi $\mathrm{SS}$, et al. EWSR1 rearrangement is present in a subset of myoepithelial tumors of salivary glands with variable morphology and does not correlate with clinical behavior. Ann Diagn Pathol. 2017;28:19-23.

17. Rekhi B, Sable M, Jambhekar NA. Histopathological, immunohistochemical and molecular spectrum of myoepithelial tumours of soft tissues. Virchows Arch. 2012;461(6):687-697.

18. Gleason BC, Fletcher CD. Myoepithelial carcinoma of soft tissue in children: an aggressive neoplasm analyzed in a series of 29 cases. Am J Surg Pathol. 2007;31(12):18131824.

19. Moussaly E, Nazha B, Kedia S, Chang Q, Forte F. Soft Tissue Myoepithelial Carcinoma of the Neck with Spinal Invasion. Rare Tumors. 2016;8(3):6485.

20. Miettinen M, Sobin LH, Sarlomo-Rikala M. Immunohistochemical spectrum of GISTs at different sites and their differential diagnosis with a reference to CD117 (KIT). Mod Pathol. 2000;13(10):1134-1142.

21. Agaram NP, Chen HW, Zhang L, Sung YS, Panicek D, Healey JH, Nielsen GP, et al. EWSR1-PBX3: a novel gene fusion in myoepithelial tumors. Genes Chromo- somes Cancer. 2015;54(2):63-71.

22. Antonescu CR, Zhang L, Chang NE, Pawel BR, Travis W, Katabi N, Edelman M, et al. EWSR1-POU5F1 fusion in soft tissue myoepithelial tumors. A molecular analysis of sixty-six cases, including soft tissue, bone, and visceral lesions, showing common involvement of the EWSR1 gene. Genes Chromosomes Cancer. 2010;49(12):11141124.

23. Skalova A, Weinreb I, Hyrcza M, Simpson RH, Laco J, Agaimy A, Vazmitel M, et al. Clear cell myoepithelial carcinoma of salivary glands showing EWSR1 rearrangement: molecular analysis of 94 salivary gland carcinomas with prominent clear cell component. Am J Surg Pathol. 2015;39(3):338-348.

24. Antonescu CR, Zhang L, Shao SY, Mosquera JM, Weinreb I, Katabi N, Fletcher CD. Frequent PLAG1 gene rearrangements in skin and soft tissue myoepithelioma with ductal differentiation. Genes Chromosomes Cancer. 2013;52(7):675-682.

25. Huang SC, Chen HW, Zhang L, Sung YS, Agaram NP, Davis M, Edelman M, et al. Novel FUS-KLF17 and EWSR1-KLF17 fusions in myoepithelial tumors. Genes Chromosomes Cancer. 2015;54(5):267-275.

26. Urbini M, Astolfi A, Indio V, Tarantino G, Serravalle S, Saponara M, Nannini M, et al. Identification of SRF-E2F1 fusion transcript in EWSR-negative myoepithelioma of the soft tissue. Oncotarget. 2017;8(36):60036-60045.

27. Mahdi Y, Zouaidia F, Zouhair A, Azouz M, Znati K, Jahid A, Berrada MS, et al. Combined myoepithelial carcinoma and myoepithelioma in soft tissue: a case report and review of the literature. J Med Case Rep. 2014;8:317.

28. Tayal S, Suri V, Misra MC, Ray R. Myoepithelial carcinoma of soft tissue: a case report. Indian J Pathol Microbiol. 2007;50(4):761-763. 